\title{
Renal Replacement Therapy
}

National Cancer Institute

\section{Source}

National Cancer Institute. Renal Replacement Therapy. NCI Thesaurus. Code C126400.

Treatments that substitute for the blood-filtering function of the kidneys. It includes dialysis (hemodialysis or peritoneal dialysis), hemofiltration, and hemodiafiltration. Kidney transplant may also be considered a renal replacement therapy. 